\title{
Delayed symptoms and death after minor head trauma with occult vertebral artery injury
}

\author{
R N Auer, J Krcek, J C Butt
}

\begin{abstract}
Head injury without loss of consciousness is seldom accompanied by grave complications. We report the case of an 18 year old cyclist who was struck by a car in a minor road traffic accident, suffered minor head injury without loss of consciousness, and died unexpectedly seven weeks later with vomiting and coma. Necropsy revealed an expanding cerebellar infarct and vertebral artery thrombosis, superimposed on an old dissecting intramural haematoma of the right vertebral artery in the atlantoaxial region. Vertebrobasilar occlusion after minor head trauma, hyperextending or rotating neck injury, or neck manipulation is commonest in young people. Occult ligamentous injury to the cervical spine after trauma may be a contributing factor to the pathogenesis of vertebral artery damage after injury to the neck.
\end{abstract}

(F Neurol Neurosurg Psychiatry 1994;57:500-502)

Head injury does not usually cause death unless there are indicators of severe head trauma, such as depression or loss of consciousness. The torsion or hyperextension of the neck accompanying even minor head injury, however, may cause damage to the vertebral artery, as indicated by the following case.

\section{Case report}

An athletic 18 year old was involved in a motor vehicle accident on 2 February 1991. While riding his bicycle, he was struck by a car. He was thrown on to the bonnet of the vehicle and rolled up to the windscreen. There was no loss of consciousness. After assessment at an emergency room 20 minutes later, he was discharged with a diagnosis of fracture of the tibia, below the tibial plateau. No head trauma could be recalled from the accident, but in view of a minor laceration on his head, he and his mother were given a head injury protocol to take home. There was no depression of consciousness over the next 24 hours.

The boy continued to be heavily involved in body building, cycling, and athletics. Three days after the accident he began to complain of headaches to his school friends. Episodes of transient global amnesia occurred, during which he would suddenly forget where he was, and what he was doing. These memory lapses were never accompanied by collapse or weakness. He complained of a persistent sore neck.

On 28 March 1991, he was difficult to arouse, and awoke with a frontal headache. He needed help dressing; his mother describing this as "dressing a baby". He stayed away from school, slept most of the day, and vomited increasingly. By early evening, alteration in affect was noted. He was taken to hospital in a drowsy but rousable state. Over the next hour he became progressively rigid and cyanotic, then unresponsive to verbal stimuli or urinary catheter insertion. Brain stem reflexes disappeared. Cardiac arrhythmias appeared, consisting of supraventricular tachycardia and ventricular bigeminy. A CT scan revealed a density in the cerebellum and secondary hydrocephalus. He was apnoeic to a $\mathrm{PCO}_{2}$ of $62 \mathrm{mmHg}$ after three minutes and was declared brain dead the following morning, 29 March 1991.

\section{NECROPSY}

Necropsy revealed a recent haemorrhagic infarct in the right cerebellar hemisphere, with oedema, tonsillar herniation, and medullary compression. A clot was present in the right vertebral artery. The cerebral hemispheres showed no gross abnormality other than acute dilatation of all ventricles. The neck was extensively dissected. No frank haemorrhages, either recent or old, were seen on visual inspection of the ligaments of the atlas or axis.

Neuropathological examination of the brain showed numerous microscopic infarcts symmetrically distributed in the vertebrobasilar territory of supply, including the occipital lobes, hippocampi, thalamus, and hypothalamus. Their sizes ranged from tens of microns to a few hundred microns in diameter, with cyst formation in the larger infarcts. Vessels showing evidence of old occlusions and residual thrombi were numerous; occlusive fibrointimal hyperplasia and deposition of intralumenal and perivascular iron were seen.

The right vertebral artery was subserially sectioned from its location in the foramen transversarium of $\mathrm{C} 2$ to the vertebrobasilar junction. Microscopical examination revealed old intramural dissection in the portion of the vessel in the atlantoaxial region. The artery 
Figure 1 Magnification of the wall of the injured artery, with the lumen at the top. Progressing outwards concentrically, the photograph shows fibrointimal hyperplasia (FIH), the internal elastic lamina (IEL), the smooth muscle and elastic lamellae of the tunica media (MED), the external elastic lamina (EEL), lighter staining granulation tissue (GR), and the adventitia (ADV). The granulation tissue, in capillaries, contains iron in macrophages, variably distributed along the circumference and the length of the dissection. Trichrome-elastin stain. addition to fibroblasts and bar $=200 \mu \mathrm{m}$.

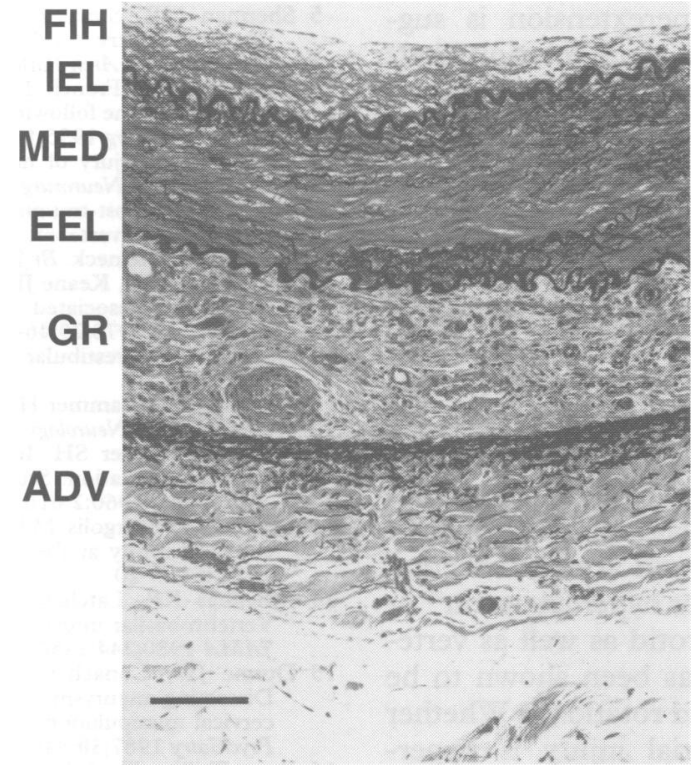

showed fibrointimal hyperplasia, but stenosis approached maximally $20 \%-30 \%$ of the lumen. The outer third of the tunica media contained fibrovascular granulation tissue, splitting the external elastic lamina from inner concentric laminae and smooth muscle (fig 1).

The dissection tapered in extent proceeding superiorly from the atlantoaxial region. At the origin of the posterior inferior cerebellar artery, however, the vessel was encased in dense fibrous tissue, enclosing the nervi vasorum. Sections through this region revealed subperineurial haemorrhage in the nervi vasorum (fig 2) of the vertebral artery. At this level acute neutrophilic inflammation was also seen within the tunica media and the areas of fibrointimal hyperplasia, extending up to defects in the internal elastic lumina and endothelium. Special stains for bacteria or fungi were negative.

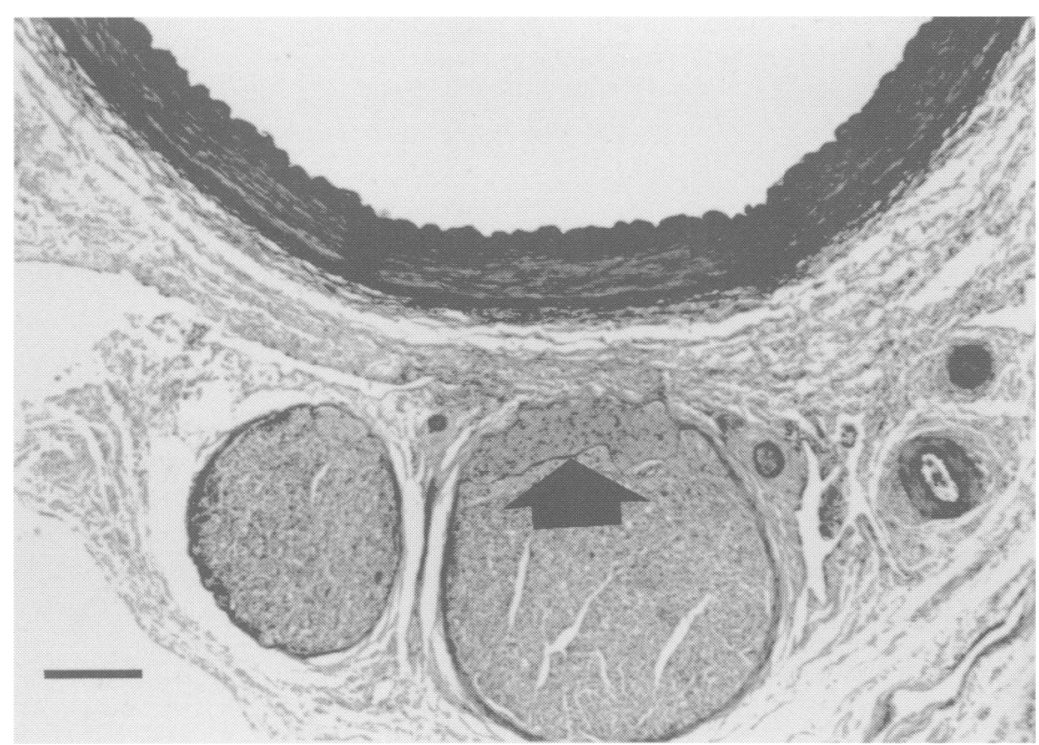

Figure 2 Posterior inferior cerebellar artery near the site of origin and two nervi vasorum. One of the nervi vasorum shows recent hemorrhage (arrow) beneath the perineurium and there is thrombosis of the vasa vasorum (right centre). The intraluminal thrombus has been displaced by postmortem angiography. Trichrome-elastin stain; bar $=100 \mu \mathrm{m}$.

\section{Discussion}

The patient died seven weeks after a minor head injury and showed evidence of both early and delayed injury to the vertebral artery. The delayed death was due to thrombosis of the vertebral artery and cerebellar infarction, and oedema and brainstem compression. Evidence of remote trauma consisted of a healing intramural dissecting haematoma of the tunica media of the right vertebral artery, fibrointimal hyperplasia, and numerous old, resorbing thromboemboli and cystic infarcts in the vertebrobasilar territory. Evidence of recent trauma to the vertebral artery included subperineurial haemorrhage, acute neutrophilic inflammation in the tunica media near the origin of the posterior inferior cerebellar artery, and recent thrombosis of the vertebral artery itself and its vasa vasorum.

The notion of occult ligamentous injury to the neck is supported by the boy's complaints of neck pain, and by the finding in a large series that such occult trauma to the cervical spine is more common than is generally appreciated in traffic accident victims. ${ }^{1}$ A series of 12 patients with either unilateral or bilateral facet joint dislocations after motor vehicle accidents revealed vertebral artery occlusions in nine patients. ${ }^{2}$ The average age was 38.8 years and no patient in the series was older than 50. Even without occult ligamentous trauma the young neck, with its greater mobility, is prone to vertebral artery injury when manipulated, indicated by the young age of most chiropractic and exercise induced injuries to the vertebral artery. ${ }^{3-5}$ It was verified that the boy did not visit a chiropractor after his injury despite the complaints of a sore neck. The final mechanism of death in injury to the vertebral artery is often oedematous cerebellar infarction with brainstem compression. ${ }^{6-8}$

At the microscopic level, the mechanism of the delayed thrombosis of the vertebral artery in the present case was likely endothelial injury superimposed on a severely injured vertebral artery with extensive fibrointimal hyperplasia and old medial dissection. The added complication of lax, injured ligaments in the neck may have played an additional part. An injured vertebral artery with a circumferential, healing dissection is likely to be prone to damage by even normal neck rotation and extension movements, in addition to the athletic movements made by this young man during the course of pursuing his varied sports. Case 1 of Devereaux et al ${ }^{9}$ showed an old vertebral artery dissection eight years after a road traffic accident, with death due to recent cerebellar and brainstem infarction.

At the gross level, the mechanism of injury to the vertebral artery in the present case may involve compression of the artery due to either lateral rotation or hyperextension. Lateral rotation of the head has been shown to cause vertebral artery obstruction, ${ }^{10-13}$ with subsequent dissection ${ }^{14-17}$ of the artery and occasionally pseudoaneurysm formation. ${ }^{18} 19$ Hyperextension may also lead to dissection of the vertebral artery. ${ }^{20-22}$ The mechanism of 
rotation rather than hyperextension is suggested in the present case, neck rotation likely occurring as the boy rolled over the bonnet of the car up on to the windscreen.

Local anatomical features unique to the atlantoaxial region $^{3}$ are often invoked to explain vertebral artery injury after head or neck trauma involving neck hyperextension or rotation. It is true that the local anatomy of the sharply turning vertebral arteries emerging from the foramena transversaria of the cervical vertebrae makes them vulnerable to occlusion with head rotation, as has been demonstrated at necropsy ${ }^{10-12}$ and angiographically. ${ }^{13}$ This cannot explain all cases of such damage, however, as carotid artery dissection may also occur after either hyperextension ${ }^{23}$ or rotation. ${ }^{24}$ Moreover, carotid as well as vertebral artery blood flow has been shown to be sharply reduced with head rotation. ${ }^{12}$ Whether the mechanism of arterial injury is hyperextension or lateral rotation, the present case illustrates the vulnerability of the young neck and especially the vertebral arteries to injury, even in cases of minor head trauma. Death may occur a considerable time after the injury due to occult vertebral artery damage in the form of a dissecting haematoma and its consequences.

1 Bucholz RW, Burkhead WZ, Graham W, Petty C. Occult cervical spine injuries in fatal traffic accidents. $\mathscr{F}$ Traum 1979;19:768-71.

2 Louw JA, Mafoyane NA, Small B, Neser CP. Occlusion of the vertebral artery in cervical spine dislocations. $\mathcal{F}$ Bone foint Surg 1990;72:679-81.

3 Frisoni GB, Anzola GP. Vertebrobasilar ischemia after neck motion. Stroke 1991;22:1452-60.

4 Phillips SJ, Maloney WJ, Gray J. Pure motor stroke due to vertebral artery dissection. Can $f$ Neurol Sci 1989; 16:348-51.
5 Sherman MR, Smialek JE, Zane WE. Pathogenesis of vertebral artery occlusion following cervical spine manipulation. Arch Pathol Lab Med 1987;111:851-3.

6 Suechting RI, French LA. Posterior inferior cerebellar artery syndrome following a fracture of the cervical vertebra. $₹$ Neurosurg 1955;12:187-9.

7 Carpenter S. Injury of neck as cause of vertebral artery thrombosis. F Neurosurg 1961;18:849-53.

8 Murray DS. Post-traumatic thrombosis of the internal carotid and vertebral arteries after non-penetrating carotid and vertebral arteries after non-pent
injuries of the neck. Brf Surg 1957;44:556-61.

9 Devereaux MW, Keane JR, Davis RI. Automatic respiratory failure associated with infarction of the medulla. tory failure associated with

10 DeKlyn A. On vestibular nystagmus. Confin Neurol 1939; 2:857-62.

11 Tatlow WFT, Bammer HG. Syndrome of vertebral artery compression. Neurology 1957;7:331-40.

12 Toole JF, Tucker SH. Influence of head position upon cerebral circulation. Studies on blood flow in cadavers. Arch Neurol 1960;2:616-23.

13 Barton JW, Margolis MT. Rotational obstruction of the vertebral artery at the atlantoaxial joint. Neuroradiology 1975;9:117-20.

14 Schellhas KP, Latchaw RE, Wendling LR, Gold LH. Vertebrobasilar injuries following cervical manipulation. ¥AMA 1980;244:1450-3.

15 Dunne JW, Conacher GN, Khagure M, Harper CG. Dissecting aneurysms of the vertebral arteries following cervical manipulation: a case report. $\mathcal{I}$ Neurol Neurosurg Psychiatry 1987;50:349-53.

16 Pryse-Phillips $W$. Infarction of the medulla and cervical cord after fitness exercises. Stroke 1989;20:292-4.

17 Mehalic T, Farhat SM. Vertebral artery injury from chiropractic manipulation of the neck. Surg Neurol 1974; 2:125-9.

18 Davidson KC, Weiford EC, Dixon GD. Traumatic vertebral artery pseudoaneurysm following chiropractic manipulation. Radiology 1975;115:651-2.

19 Sherman DG, Hart RG, Easton JD. Abrupt change in head position and cerebral infarction. Stroke 1981;12. $2-6$.

20 Simeone FA, Goldberg HI. Thrombosis of the vertebral artery from hyperextension injury to the neck. artery from hyperextension

21 Nagler W. Yertebral artery obstruction by hyperextension of the neck. Arch Phys Med Rehabil 1973;54:237-240.

22 Six EG, Stringer WL, Cowley AR, Davis CH Jr. Posttraumatic bilateral vertebral artery occlusion: case report. F Neurosurg 1981;54:814-7.

23 Trosch RM, Hasbani M, Brass LM. "Bottoms up" dissection. N Engl $f$ Med 1989;320:1564-5.

24 Beatty RA. Dissecting haematoma of the internal carotid artery following chiropratic cervical manipulation f Trauma 1977;17:248-9. 